\title{
SEIZURES IN CHILDREN WITH LOW GRADE GLIOMA
}

\author{
Ostwal Piyush $^{1}$, Shanbhag Nandan ${ }^{2}$ \\ Correspondence: piyush.ostwal@gmail.com \\ ${ }^{l}$ Neurologist, Department of Neurology, Bahrain Specialist Hospital, Bahrain \\ ${ }^{2}$ Chief Consultant Oncologist Mount Saint John's Medical Centre, St John's, Antigua and Barbuda
}

Article History:

Received: July 15, 2020

Accepted: December 1, 2020

Published: January 1, 2021

\section{Cite this as:}

Piyush $O$, Nandan S. Seizures in children with low grade glioma. Malang Neurology Journal; 2021.7:60-65.

http://dx.doi.org/10.21776/ub.mnj .2021 .007 .01 .12

\section{ABSTRACT}

Seizures are a common presentation of pediatric brain tumors. The incidence of pediatric brain tumor (Age 0-19 years) ranges from 1.12-5.26 cases per 100,000 persons. Low grade gliomas are an important subgroup of pediatric brain tumors causing epilepsy. Low-grade gliomas are largely slowgrowing tumors and the manifestations are dependent on age, location, tumor type, size of tumor and rate of tumor growth. Seizures have been reported in up to $38 \%$ of children with supratentorial tumors. The tumors are identified when work up of patients for epilepsy includes electrophysiological and imaging studies. The primary treatment modality remains surgical excision. Antiepileptic medications are used for control of seizures. Subsequent histopathological diagnosis is important for prognostication. The tumors commonly associated with long-term epilepsy in various studies were ganglioglioma, dysembryoplastic neuroepithelial tumor, pilocytic astrocytoma and pilocytic xanthoastrocytoma. The outcome of surgery with regards to seizure control is generally good. Though concomitantly antiepileptic medications will be needed for most of them. An attempt is made in this review to summarize the epidemiology, clinical features, pathology and treatment aspects of pediatric low grade gliomas presenting with seizures. conclusion.

Keywords: Seizures, Low grade glioma, Pediatric epilepsy, brain tumor

\section{Introduction}

Tumors are a form of cancer resulting from uncontrolled growth of cells in a solid organ. Cancer is prevalent globally, and although with growing health apprehension and therapeutic development, cancer mortality rates are still alarming. Moreover, a recent report in 2017 estimated 23,800 new cases of brain and central nervous system (CNS) tumors out of 1,688,780 new cases estimated in the United States alone. Despite the low prevalence as compared with other cancers, the statistical rate of mortality from glioma and other related brain tumors is estimated to be around $70 \%$ in the year 2017 alone. Glioma, which refers to tumors of glial cell origin, is the most common type of central CNS tumor and constitutes more than $30 \%$ of all primary brain and CNS malignant tumors. According to the World Health Organization, gliomas are classified into four different classes with different grades: astrocytoma (Grade I - II), anaplastic astrocytoma (grade III), oligodendroglioma, ependymoma, mixed glioma, and glioblastoma multiforme (GBM) (grade IV). Among oligodendrogliomas, ependymomas, mixed gliomas, and glioblastoma multiforme, GBM is known to be the most lethal form of glioma with the worst prognosis. ${ }^{1,2}$

Primary brain tumors are the most common pediatric solid tumors. Pediatric gliomas represent a diverse group of tumors of varying histologies, grades, and genetics. Broadly, these tumors are divided into two major categories: pediatric low-grade (PLGG) and high-grade (PHGG) gliomas. Traditionally, gliomas have been diagnosed solely on histologic criteria; however, because morphologic overlap occurs frequently, there remains a need for objective data to promote diagnostic accuracy and support prognostic and therapeutic decision-making., 3 The diagnosis of brain tumor eventuates in an immense psychological trauma and social economic burden for the caregivers. The management of these tumors requires highly specialized personnel in a well-equipped dedicated tertiary care setup.

Seizures are a common presentation of pediatric brain tumors. There is social stigma associated with the disease and there are practical issues in the daily life faced by the patient. Seizures by themselves pose a major diagnostic and therapeutic challenge in children. The difficulty starts with ascertaining the true description of the event and differentiating it from other mimics. The next obstacle is searching for focus and etiology. With the availability of neuroimaging the diagnosis and treatment has become more precise and evidence-based.

So when brain tumors and seizures are present together in children the problem becomes multifaceted and holistic approach is needed to achieve an optimum result. The treating team needs to fall back on experience and evidence for choosing an appropriate strategy to redress both tumor and seizures.

Low grade gliomas are an important subgroup of pediatric brain tumors causing epilepsy. There is a great heterogeneity in the studies that examine the relationship between seizures and pediatric low-grade gliomas. The 
information obtained from studies in adults cannot be extrapolated to pediatric age group. One set of studies evaluated patients with refractory epilepsy who are later found to have tumor.5,6,7 Another approach involves description of presentations of brain tumors with seizures being one of them. ${ }^{8,9}$ By virtue of a great amount of biological difference between different tumor types the clinical presentation can be very variable. The aim of treatment also varies from control of seizures to oncologic aspects like recurrence and tumor growth. Some of the tumors remain benign and cause morbidity due to the recurrent seizures. Other tumors have malignant potential and require additional treatment with chemotherapy and radiotherapy. These additional treatments have their own effect on control of seizures as well as potential contribution to epileptogenicity. The available evidence for choosing the management strategy in pediatric low grade glioma with seizures is patchy and does not hold good for a given clinical scenario. The data available for individual tumor types with regards to their presentation, natural history, appropriate treatment and prognosis is not easily available. There are practical difficulties with data collection in the disease which runs over years. These studies require coordinated efforts between pediatric neurologists, pediatric neurosurgeons and pathologists over extended periods of time. The pathological classification of brain tumors is ever evolving and comparison of studies done far apart in time may not be meaningful. The prospective studies are virtually nonexistent and data available from retrospective analysis has its own limitations. In this paper we will review the association between seizures and low-grade gliomas in pediatric age group.

\section{Epidemiology and Clinical Features}

The incidence of pediatric brain tumor (Age 0-19 years) ranges from 1.12-5.26 cases per 100,000 persons with lowest being in Middle East region of Kuwait and highest in United States. The incidence is highest in 0-4 year age group and lowest in 10 to 14 year age group. However incidence of individual tumor subtypes can peak in different age groups. ${ }^{10}$

Low grade glioma refers to tumors of glial origin categorized under World Health Organization (WHO) grade 1 and 2. ${ }^{11}$ The types of pediatric LGG which are relevant to this discussion and their tumor grade are included in Table 1.

The incidence of low-grade brain tumor in the group below 20 years in United States is estimated to be 1.7 per 100,000 population. $^{12}$ Pilocytic astrocytoma is one of the most common among pediatric brain tumors counting for $17 \%$ of cases in 0 to 14 year age group. ${ }^{10}$

Pediatric brain tumors can present with symptoms attributable to elevated intracranial pressure (headache, nausea, vomiting, papilledema), neurological deficits (incoordination, abnormal gait, weakness), seizures and nonspecific symptoms (change in behavior, school performance, irritability). Low-grade gliomas are largely slow-growing tumors and the manifestations are largely dependent on age, location, tumor type, size of tumor and rate of tumor growth. The 10- and 20-year overall survival of $87 \%$ and $82 \%$, respectively was reported in a study of low grade glioma in children. So with this good survivor neurologists are more likely to see such patients for other comorbidities like epilepsy. ${ }^{8}$

Table 1. Low grade gliomas and their WHO tumor grade

\begin{tabular}{lc}
\hline \multicolumn{1}{c}{ Tumor Subtype } & Tumor Grade \\
\hline Astrocytic tumors & \\
Subependymal giant cell astrocytoma & I \\
Pilocytic astrocytoma & I \\
Pilomyxoid astrocytoma & II \\
Diffuse astrocytoma & II \\
Pleomorphic xanthoastrocytoma & II \\
\hline Oligodendroglial and oliogoastrocytic tumors & \\
Oligodendroglioma & II \\
Oligoastrocytoma & II \\
\hline Neuronal and mixed neuronal-glial & \\
Ganglioglioma & I \\
Desmoplastic infantile ganglioglioma & I \\
Dysembryoplastic neuroepithelial tumor & I \\
Central neurocytoma & II \\
\hline
\end{tabular}

Seizures are an important manifestation of brain tumor. In a meta-analysis of presenting signs and symptoms of pediatric brain tumors seizures were present in $13 \%$ of children at diagnosis. In the same review the frequency of seizures during the course of illness for supratentorial tumors stood at $38 \% .^{9}$ In a study with large cohort of pediatric brain tumor follow-up patients, seizures were reported in $24 \%$ children of which $10 \%$ children already had it by the time tumor diagnosis was made and in the remaining $14 \%$ they manifested during course of follow up. Some of these children had onset of seizures even more than two years after the completion of treatment. ${ }^{13}$ In a study from Israel of children with brain tumor, seizures were present in $15.5 \%$ children. For almost half them seizures were the sole symptom. ${ }^{14}$ In another study where only low grade gliomas were included the seizures were seen in $38 \%$ of children. The cumulative incidence of seizures continued to rise with follow-up reflecting a persistent risk of developing epilepsy in survivors of low grade glioma. Among the survivors who developed a neurological deficit later, a large percentage (34\%) developed epilepsy beyond 5 years from diagnosis. ${ }^{8}$ Thus seizures can continue to appear and surprise patients, caregivers and physicians long after initial disease process is treated. This definitely adds an element of uncertainty, restrictions on lifestyle and future planning for children growing up with history of brain tumors.

Histopathological nature of tumor has a considerable impact on the association with seizure potential in children. In a study glioneuronal tumors $(17 / 21,81 \%)$, high grade glioma $(5 / 7,71.4 \%)$, and oligodendroglioma (4/5, 80\%) were most frequently associated with seizures as against medulloblastoma, germ cell tumor, atypical teratoid rhabdoid tumor, and pineal tumors (pineocytoma and pineoblastoma). ${ }^{13}$ Low grade tumors are postulated to pose a high risk of seizures compared to high grade tumors. Ninety percent of tumors in a study were low grade among children presenting with seizures. The tumor types reported in this study were 12 ganglioglioma, 12 pilocytic astrocytomas, 10 astrocytomas and 7 dysembryoplastic neuroepithelial tumor (DNET). ${ }^{14}$ Low grade tumors predominated in another study of children with refractory 
epilepsy operated for brain tumor where all the tumors were low-grade except one which was grade 3 anaplastic oligodendroglioma. DNET and ganglioglioma constituted one third each. Among others oligodendroglioma was most frequently seen $(11 \%){ }^{5}$

Luyken et al have described long-term epilepsy associated tumors (LEAT) in their series of patients with supratentorial tumors operated for refractory epilepsy. This was a mixed age group cohort and children were not analyzed separately. The tumors typically identified to be associated with long-term epilepsy were ganglioglioma, DNET, pilocytic astrocytoma and pilocytic xanthoastrocytoma WHO grade I. These are benign tumors and have excellent prognosis. There was another group described which includes diffuse astrocytomas WHO grade II and oligodendrogliomas WHO grade II which have a lower survival but are encountered in patients with longterm epilepsy. ${ }^{15}$

In a cohort of children with brain tumors who underwent epilepsy surgery the histopathology revealed $39(46.4 \%)$ patients with gliomas, 20 (23.8\%) with DNETs, 14 (16.7\%) with gangliogliomas, and $11(13.1 \%)$ with other etiologies. $^{16}$

From a cohort of 50 children who underwent posterior cortex (involving the occipital and/or parietal lobe and sometimes extending to the posterior border of the temporal lobe) epilepsy surgery, 16 patients (32\%) were identified to have benign brain tumors. Even in the posterior cortex large majority was constituted by DNET (10/16) and ganglioglioma (4/16). There was only one pilocytic astrocytoma. ${ }^{6}$ In a study of temporal lobe low grade tumors with epilepsy the tumor types reported were pilocytic astrocytoma (20/41), ganglioglioma (12/41), low-grade oligodendroglioma (6/41) and DNET (3/41). ${ }^{17}$

Among 361 children who were followed after diagnosis of LGG most common was pilocytic astrocytoma (63\%). This study did not include glioneural tumors. The tumors included under low grade glioma were grade 1 astrocytoma (pilocytic) or grade 2 astrocytoma (including pilomyxoid fibrillary astrocytoma, oligoastrocytoma, oligodendroglioma, or low-grade astrocytic tumors not otherwise specified). So in this series a large proportion of tumors typically associated with long-term epilepsy (LEAT) were not part of analysis. The pilocytic astrocytoma was majorly located in the diencephalon (41\%) or the cerebellum (26\%). And less commonly it was located in the cerebral hemispheres $(15 \%)$ followed by brainstem (13\%) and spinal cord (5\%). In overall study also hemispheric location of tumor was seen in $15 \%$ patients. The incidence of seizure was highest in tumors located in cerebral hemispheres $(76 \%)$, but other tumor locations of posterior fossa and hypothalamus/ chiasma contributed to seizures as well. In multivariate analysis also supratentorial tumor location was the only factor which was significant risk factor. ${ }^{8}$ Even a higher figure of $94.4 \%$ of tumors being supratentorial in children presenting with seizures has been reported. $^{14}$ A large proportion of tumors being infratentorial, this difference is highly relevant in pediatric age. In a study $52 \%$ of children with cortical tumors resulted in seizure, whereas only $9 \%$ of infratentorial tumors and $18 \%$ of midline tumors experienced seizures. ${ }^{13}$ As high as $96 \%$ of tumors operated for refractory epilepsy were supratentorial in one study. ${ }^{5}$ So where as presence of seizures points strongly to cortical tumor, it does not rule out infratentorial tumors.

Among cortical locations temporal lobe tumors are more often responsible for seizures compared to other lobes. In a study 41/48 patients with temporal lobe low-grade tumor in pediatric age group had seizure as the presenting symptom. Nine of these patients with seizures had refractory epilepsy. ${ }^{17}$ In other studies also similar propensity of temporal lobe tumors to develop epilepsy has been described. ${ }^{14}$ The typical LEATs described by Luyken were most often present in temporal lobe (83 \%). Even astrocytomas associated with epilepsy were present more often in temporal lobe (68\%) compared to frontal lobe (21 $\%) .{ }^{15}$ Among supratentorial locations temporal lobe (59.3) was most common followed by parietal lobe (25.9) in another study of children with refractory epilepsy operated for brain tumor. ${ }^{5}$ Temporal lobe $(51.9 \%)$ and frontal lobe $(31.5 \%)$ predominated in another study on seizures in children with brain tumors. ${ }^{14}$ Tumor recurrence is another risk factor for seizure appearance or recurrence during follow up. ${ }^{13}$ Other variables like age at diagnosis was not associated with later risk of epilepsy. ${ }^{8}$

The seizure frequency in pediatric brain tumors can be highly variable. The frequency depends on tumor pathology and location of tumor. The inclusion criteria of the studies are highly relevant when studying this particular aspect because enrollment from refractory epilepsy cohort typically will have high frequency of seizures. In a cohort of children with brain tumors who underwent epilepsy surgery, half of the patient's had daily seizures and one third had weekly seizures. The high frequency of seizures in this series is obviously related to the patient selection reflecting the patient attending the refractory epilepsy clinic are more likely to have more frequent seizures. ${ }^{16}$ Similar seizure frequency preoperatively in children with refractory epilepsy and brain tumor was reported as daily in $48 \%$, weekly in $41 \%$ and monthly in $11 \%$. $^{5}$

The neuronal networks in developing brain of children is constantly evolving and so semiology of seizures is not typical of adult types and varies with age of the child. As expected focal seizures originating from tumor focus are common. Complex partial seizures are the most common seizure types reported in children with low-grade brain tumors. In a study of children presenting with brain tumors complex partial seizure was present in $42.6 \%$, partial with generalized in $24.1 \%$ and simple partial seizures in $18.5 \%$. Eight children in this group had generalized seizures. ${ }^{14}$

In a study of children with epilepsy with low grade glial tumors in temporal lobe, complex partial seizure was the most common seizure semiology present in 30 of 41 patients. Four of these patients with complex partial seizures had secondary generalization. Simple partial seizures were present in only two patients. Generalized tonic clonic seizures were seen in four patients and combine seizure type in five patients. ${ }^{17}$ In another study of refractory epilepsy in children with tumors the seizure type complex partial seizures were present in all the children, a third of whom had secondary generalization. The proportion of children with simple partial seizures and generalized tonic clonic seizures was rather small $\left(7 \%\right.$ each). ${ }^{5}$ So presence of focal seizures should arise suspicion of brain tumor in 
children. However presentation with generalized seizures does not rule out brain tumor as a cause of seizures.

\section{Pathogenesis}

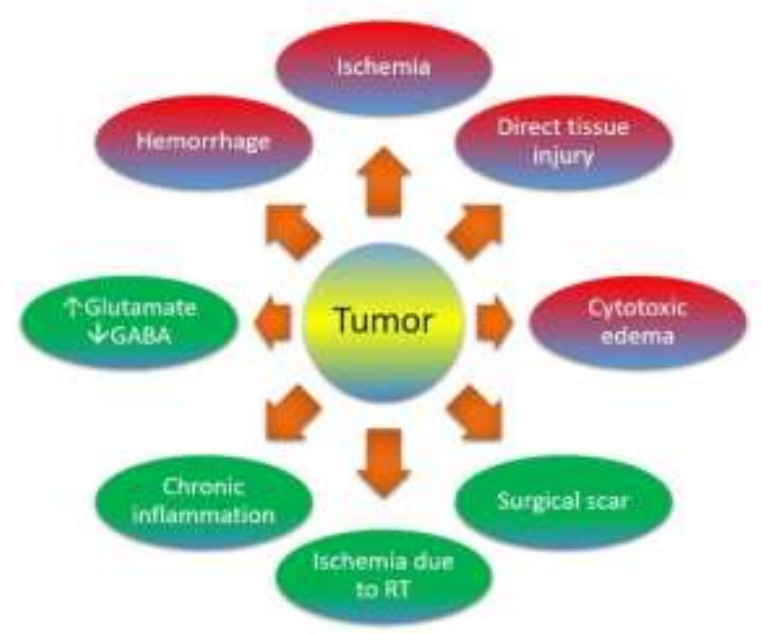

Figure 1. The diagram depicts the brain tumor related factors which are relevant in the pathogenesis of seizures. Those in red bubbles are more often associated with high grade gliomas.

GABA- Gamma aminobutyric acid, RT - radiotherapy

There is a large variation in histopathological characteristics of tumors lumped under the category of low grade glioma. Regardless of subtype, tumors located in temporal lobe are more apt at recruiting epileptogenic networks. For high grade tumors, the ischemia, hemorrhage, direct tissue injury and cytotoxic edema appear to be more important factors leading to seizures (Figure 1). Whereas slow growth of low grade brain tumors may account for the focal evolving changes in the brain parenchyma which can contribute to epileptogenesis. The tumors can alter concentration of neurotransmitters such as glutamate and GABA contributing to increased excitability and seizures. Some of the molecular markers found to be influencing seizures in low-grade gliomas are IDH $1 / 2$ mutations, BRAF V600E mutations, overexpression of nuclear protein Ki-67, high RINT1 expression, Low expression of very large G-protein-coupled receptor-1 (VLGR) 1 and dysregulation of miR-128 expression. ${ }^{7} \mathrm{~A}$ better understanding of the relationship between molecular pathologies such as IDH/TERTP mutations and seizures in LGG patients has been uncovered as having a very important clinical and social value for setting therapeutic regimens and improving the life quality of LGG patients. Some researchers have actively investigated the potential relationship between IDH/TERTP mutations and preoperative seizures in patients with LGG and to reveal whether the IDH/TERTP mutations are related to the onset of seizures and seizure type. ${ }^{2,18}$ Low-grade tumors have been known to be associated with adjacent cortical dysplasia which can independently be the cause of seizures even after primary tumor has been resected. Neurosurgical intervention can alter the peritumoral brain parenchyma which may become focus for epilepsy in future. Radiotherapy induces late vasculopathic changes causing ischemia and predisposition to seizures. ${ }^{13}$ The role of chronic inflammation in the peritumoral tissue has also been implicated in epileptogenesis. ${ }^{5}$

\section{Diagnosis}

If seizures are the presenting symptoms the clinician needs to differentiate it from a number of paroxysmal attacks which are nonepileptic like syncope, aura of migraine, vertigo, breath holding spells, movement disorders, cataplexy, self-stimulatory behavior, sleep walking and night terrors. A detailed reliable history from an eyewitness or home video record are most helpful initially. In the absence of other signs and symptoms, the first attack of generalized seizure may not lead to further investigation and diagnosis of tumors may be delayed. When neuroimaging is done for evaluation of seizure, it may reveal tumor. EEG will help in detection of epileptiform discharges arising from seizure focus. When recruited from refractory epilepsy clinic focal EEG abnormality corresponding to tumor location was seen in majority of children (85\%). EEG was normal in only one child out of studied cohort. ${ }^{5}$ In a study of 48 children with seizure and brain tumor who underwent EEG, it was abnormal in $62.5 \%$ patients with abnormality localizing to tumor area in $18 / 30$ cases. The most common finding was focal epileptiform pattern in 23/30 cases. One patient had generalized epileptiform activity. Focal slowing and generalized slowing were seen in 3 cases each. ${ }^{14}$ So though localizing findings are more common in EEG, still generalized abnormality does not rule out tumor as possible cause.

Further detailed noninvasive and invasive electrophysiological studies may be required for delineation of epileptogenic focus. Some centers use Electrocorticography $(\mathrm{ECoG})$ regularly for delineating the zone of resection. However the benefit of ECoG in this setting is not established. ${ }^{16}$ Resection of the tumor offers accurate histopathological diagnosis and is of therapeutic value in control of seizures due to low grade glioma.

\section{Treatment of Seizures}

Further Seizures add to the burden of morbidity in LGGs. Seizures are unpredictable, potentially life threatening and require long term therapy. Low grade glioma patients have typically longer survival and require more attention to their seizure control for a better quality of life.

There are no randomized controlled trials regarding appropriate time to start antiepileptic drugs (AED) in children with seizure and brain tumor. However first unprovoked seizures and presence of brain tumor are significant risk factors for recurrence and AEDs would be recommended in most of these children. The newer AEDs like levetiracetam and lacosamide are better tolerated and have lower systemic side effects. Also they have minimal interactions with chemotherapy drugs because they do not induce liver enzymes. Gabapentin can also be used as an add-on drug for similar benefit. The older AEDs, though efficacious, have disadvantage in terms of more side effects and interactions. Valproic acid has been seen to have an antitumor effect in vivo and in vitro, however clinical beneficial effect of this phenomena is equivocal. ${ }^{19}$ So AEDs should be individualized based on the seizure type, comorbidities, age, concurrent chemotherapy and. radiotherapy. From a refractory epilepsy group with brain tumors only a small proportion (18.5\%) was on 
monotherapy for seizures and majority required poly therapy. ${ }^{5}$ However seizures in $73 \%$ of pediatric brain tumor patients were controlled well with one medication in another study recruiting from follow up brain tumor clinic. $^{13}$ In a study where seizure control before tumor surgery was analysed, $32 \%$ of children had complete response to antiepileptic drugs, $41 \%$ patients had partial response and $27 \%$ patients had no response to antiepileptic drugs. ${ }^{14}$

This reflects wide variation in the response to therapy and need for individualization of treatment. Children should be followed up periodically and medications should be reviewed with regards to side effects and control of seizures. Surgery remains primary modality of treatment for brain tumors and in controlling epilepsy refractory to medical management. Advanced modalities like stereotaxy, intraoperative microscope, intraoperative ultrasound, the ultrasonic aspirator, evoked potentials, and advanced functional neuroimaging are being used to offer finer resection with removal of tumor and preservation of eloquent cortex.

Rate of seizure freedom following epilepsy surgery in a cohort of children with medically refractory epilepsy with brain tumor was good. Seventy five percent of children remained seizure free at 2 years following surgery. The location of the tumor in the temporal lobe was a favourable factor for good outcome. Completeness of the resection also was associated with better outcome. ${ }^{16}$ Good postoperative seizure outcome of Engel I was reported for $77.6 \%$ of children in another study. The factors favoring good outcome were low frequency of seizures preoperatively and response to antiepileptic drugs before surgery. Hemispheric location was also favorable for good seizure control following surgery compared to midline tumors. ${ }^{14}$

In another study $83 \%$ of the patients with seizures who underwent surgery had good postoperative seizure outcome being classified as Engel I. Age at seizure onset, seizure semiology and preoperative seizure duration did not have an influence on postoperative Engel score. DNET tumors exhibited relatively poor postoperative Engel score compared to others. ${ }^{17}$

Some patients with low-grade glioma may be candidates for chemotherapy such as when gross total resection cannot be achieved or if there is a recurrence. Chemotherapy has been shown to have an impact on seizure control in adult studies where combination of AEDs and temozolomide resulted in reduced frequency of seizures. ${ }^{20}$ However chemotherapy can be a cause of seizure sometimes as an adverse effect. ${ }^{21}$ Radiotherapy for pediatric low-grade gliomas is reserved for children who are not left with the option of surgery or chemotherapy. This is primarily because of long-term sequelae of brain irradiation like vasculopathy, secondary tumors, endocrine disorders and cognitive dysfunction. ${ }^{8},{ }^{13}$ Some of these may contribute to seizures later in life.

\section{Conclusion}

The brain of children is developing and is susceptible to damage from tumor, epilepsy and the treatment. Ganglioglioma, DNET, pilocytic astrocytoma and pilocytic xanthoastrocytoma WHO grade I are the tumor types frequently associated with epilepsy. The supratentorial location especially in temporal lobe is often associated with epilepsy. Specific molecular markers such as IDH/TERTP mutations found on tumors have been linked to propensity for seizure. Surgical resection of the tumor is beneficial for seizure control as well. There is lack of information about behavior of individual tumor types in children. Welldesigned prospective studies are needed to address this aspect, so that appropriate plan of management can be advised for best long-term outcome. The correlation of clinical and molecular aspects of tumors with regards to epileptogenesis is likely to open new avenues of targeted treatment of tumors and tumors related seizures.

\section{Acknowledgment}

None

\section{References}

1. Vinci M, Burford A, Molinari V, Kessler K, Popov S, Clarke M, et al. Functional diversity and cooperativity between subclonal populations of pediatric glioblastoma and diffuse intrinsic pontine glioma cells. Nature Medicine. 2018 Aug;24(8):1204-15. DOI: $10.1038 / \mathrm{s} 41591-018-0086-7$

2. Duan W, Wang L, Li K, Wang W, Zhan Y, Zhang F, et al. IDH mutations but not TERTp mutations are associated with seizures in lower-grade gliomas: Medicine. 2018 Dec;97(50):e13675.

DOI: 10.1097/MD.0000000000013675

3. Zhou W, Ke SQ, Huang Z, Flavahan W, Fang X, Paul $\mathrm{J}$, et al. Periostin secreted by glioblastoma stem cells recruits M2 tumour-associated macrophages and promotes malignant growth. Nature Cell Biology. 2015 Feb;17(2):170-82.

DOI: $10.1038 /$ ncb3090

4. Johnson A, Severson E, Gay L, Vergilio J, Elvin J, Suh J, et al. Comprehensive Genomic Profiling of 282 Pediatric Low- and High-Grade Gliomas Reveals Genomic Drivers, Tumor Mutational Burden, and Hypermutation Signatures. The Oncologist. 2017 Dec;22(12):1478-90.

DOI: $10.1634 /$ theoncologist.2017-0242

5. Bernardino MRA, Funayama C, Hamad APA, Machado H, Sakamoto A, Thome U, et al. Refractory epilepsy in children with brain tumors. The urgency of neurosurgery. Arquivos de Neuro-Psiquiatria. 2016 Dec;74(12):1008-13. DOI: $\quad$ 10.1590/0004282x20160157

6. Ramantani G, Stathi A, Brandt A, Strobl K, SchubertBast S, Wiegand G, et al. Posterior cortex epilepsy surgery in childhood and adolescence: Predictors of long-term seizure outcome. Epilepsia. 2017 Mar;58(3):412-9. DOI: 10.1111/epi.13654

7. Ertürk Çetin Ö, İşler C, Uzan M, Özkara Ç. Epilepsyrelated brain tumors. Seizure. 2017 Jan;44:93-7.

DOI: 10.1016/j.seizure.2016.12.012

8. Armstrong GT, Conklin HM, Huang S, Srivastava D, Sanford R, Ellison DW, et al. Survival and long-term health and cognitive outcomes after low-grade glioma. Neuro-Oncology. 2011 Feb;13(2):223-34.

DOI: $10.1093 /$ neuonc/noq178 
9. Wilne S, Collier J, Kennedy C, Koller K, Grundy R, Walker D. Presentation of childhood CNS tumours: a systematic review and meta-analysis. The Lancet Oncology. 2007 Aug;8(8):685-95.

DOI: 10.1016/S1470-2045(07)70207-3

10. Johnson KJ, Cullen J, Barnholtz-Sloan JS, Ostrom QT, Langer CE, Turner MC, et al. Childhood Brain Tumor Epidemiology: A Brain Tumor Epidemiology Consortium Review. Cancer Epidemiology Biomarkers \& Prevention. 2014 Dec 1;23(12):271636. DOI: 10.1634/theoncologist.2017-0242

11. Louis DN, Ohgaki H, Wiestler OD, Cavenee WK, Burger PC, Jouvet A, et al. The 2007 WHO Classification of Tumours of the Central Nervous System. Acta Neuropathologica. 2007 Jul 12;114(2):97-109. DOI: 10.1007/s00401-007-0243-4

12. SEER Cancer Statistics Review, 1975-2015 [Internet]. National Cancer Institute. Bethesda, MD; Available from: https://seer.cancer.gov/csr/1975_2015/

13. Ullrich NJ, Pomeroy SL, Kapur K, Manley PE, Goumnerova LC, Loddenkemper T. Incidence, risk factors, and longitudinal outcome of seizures in longterm survivors of pediatric brain tumors. Epilepsia. 2015 Oct;56(10):1599-604. DOI: 10.1111/epi.13112

14. Fattal-Valevski A, Nissan N, Kramer U, Constantini S. Seizures as the Clinical Presenting Symptom in Children with Brain Tumors. Journal of Child Neurology. 2013 Mar;28(3):292-6.

DOI: $10.1177 / 0883073812445786$

15. Luyken C, Blümcke I, Fimmers R, Urbach H, Elger $\mathrm{CE}$, Wiestler OD, et al. The spectrum of long-term epilepsy-associated tumors: long-term seizure and tumor outcome and neurosurgical aspects. Epilepsia.
2003 Jun;44(6):822-30. DOI: 10.1046/j.15281157.2003.56102.x

16. Fallah A, Weil AG, Sur S, Miller I, Jayakar P, Morrison G, et al. Epilepsy surgery related to pediatric brain tumors: Miami Children's Hospital experience. Journal of Neurosurgery: Pediatrics. 2015 Dec;16(6):675-80. DOI: 10.3171/2015.4.PEDS14476

17. Uliel-Sibony S, Kramer U, Fried I, Fattal-Valevski A, Constantini S. Pediatric temporal low-grade glial tumors: epilepsy outcome following resection in 48 children. Childs Nerv Syst. 2011 Sep;27(9):1413-8. DOI: $10.1007 / \mathrm{s} 00381-011-1454-5$

18. Kopinja J, Sevilla RS, Levitan D, Dai D, Vanko A, Spooner E, et al. A Brain Penetrant Mutant IDH1 Inhibitor Provides In Vivo Survival Benefit. Scientific Reports [Internet]. 2017 Dec [cited 2019 Aug 26];7(1). Available from: http://www.nature.com/articles/s41598-017-14065-w

19. Vecht CJ, van Breemen M. Optimizing therapy of seizures in patients with brain tumors. Neurology. 2006 Dec 26;67(Issue 12, Supplement 4):S10-3. DOI: 10.1212/WNL.67.12_suppl_4.S10

20. Sherman JH, Moldovan K, Yeoh HK, Starke RM, Pouratian N, Shaffrey ME, et al. Impact of temozolomide chemotherapy on seizure frequency in patients with low-grade gliomas: Clinical article. Journal of Neurosurgery. 2011 Jun;114(6):1617-21. DOI: $10.3171 / 2010.12 . J N S 101602$

21. Raisa N, Marhaendraputro EA. The side effects of chemotherapy in glioma. Malang Neurology Journal; 2019. May 2;5(2):92-7.

DOI: $10.21776 /$ ub.mnj.2019.005.02.9 